\title{
Clinicopathologic and Prognostic Significance of Carboxyl Terminus of Hsp70-interacting Protein in HBV-related Hepatocellular Carcinoma
}

\author{
Ye Jin ${ }^{1 \&}$, Li Zhou ${ }^{2 \&}$, Zhi-Yong Liang ${ }^{3}$, Ke-Min Jin ${ }^{4}$, Wei-Xun Zhou ${ }^{3}$, Bao-Cai Xing ${ }^{4 *}$
}

\begin{abstract}
Background: Many factors, including molecular ones, were demonstrated to be associated with long-term prognosis of hepatocellular carcinoma (HCC). Thus far, the expression and clinicopathologic and prognostic significance of the carboxyl terminus of Hsp70-interacting protein (CHIP) in B-type hepatitis virus (HBV)related HCC remain unknown. Materials and Methods: CHIP expression was detected by immunohistochemical staining of surgical samples from 79 patients with HCC with HBsAg positivity. In addition, correlations with clinicopathologic parameters and patient survival were evaluated. Results: It was found that positive CHIP staining was observed in tumor, but not non-tumor, tissues. High expression of CHIP was significantly related to larger tumor size, with marginally significant associations noted for presence of portal vein invasion and higher serum a-fetoprotein level. In addition, univariate analysis showed that high CHIP expression was a powerful predictor for dismal overall and disease-free survival. However, independent prognostic implications of CHIP were not proven in multivariate Cox regression test. Conclusions: CHIP is overexpressed in HBV-related HCC and is associated with unfavorable biological behavior as well as poor prognosis. However, its prognostic role needs to be further validated.
\end{abstract}

Keywords: Carboxyl terminus of Hsp70-interacting protein - B-type hepatitis virus related hepatocellular carcinoma

Asian Pac J Cancer Prev, 16 (9), 3709-3713

\section{Introduction}

Hepatocellular carcinoma (HCC) is a highly prevalent and lethal malignancy (Parkin et al., 1993; Pisani et al., 1999; Parkin et al., 2005), that is largely associated with chronic B-type hepatitis (HBV) infection (El-Serag, 2011), worldwide. Thus far, it has been well known that HCC carries poor prognosis, although some favorable results have been reported in selected patients (Poon et al., 2001; Shimozawa et al., 2004; Verhoef et al., 2004). Therefore, prognostic indicators of HCC were long of interest. It was summarized that many clinicopathologic parameters were predictive for long-term outcome of HCC (Tandon et al., 2009). Recently, more and more alterations in genes/ proteins that are involved in cell phenotypes, such as expression pattern and methylation, have been found to be associated with prognosis of this neoplasm (Mínguez et al., 2011). However, further evidence remains to be accumulated.

Carboxyl terminus of Hsp70-interacting protein (CHIP) that was first cloned in human heart (Ballinger et al., 1999), has been indicated to play important biological roles in several types of malignant neoplasm, including glioma, prostate cancer, gastric cancer, breast cancer and pancreatic cancer (Jang et al., 2011; Xu et al., 2011; Wang et al., 2013; Sarkar et al., 2014; Wang et al., 2014). Interestingly, opposite results concerning CHIP were derived from different tumor types, i.e., functioning as a potential oncogene in glioma (Xu et al., 2011) and as a tumor repressor gene (TSG) in other cancers (Jang et al., 2011; Wang et al., 2013; Sarkar et al., 2014; Wang et al., 2014). In human specimens, expression of CHIP was also shown to be a predictor of good or poor prognosis of different kinds of cancers (Jan et al., 2011; Liang et al., 2013; Wang et al., 2013; Wen et al., 2013; Wang et al., 2014). Therefore, the biological effects and prognostic significance of CHIP might be tissue-type specific. However, expression, clinicopathologic and prognostic values of CHIP in HCC have not been studied.

In the present investigation, the authors aimed to address the issues in Chinese patients with HBV-related HCC.

\section{Materials and Methods}

\section{Patients and specimens}

${ }^{1}$ Clinical Research Laboratory, ${ }^{2}$ Department of General Surgery, ${ }^{3}$ Department of Pathology, Peking Union Medical College Hospital, Chinese Academy of Medical Sciences/Peking Union Medical College, ${ }^{4}$ Key Laboratory of Carcinogenesis and Translational Research (Ministry of Education), Department of Hepatobiliary Surgery I, Peking University School of Oncology, Beijing Cancer Hospital \& Institute, Beijing, China ${ }^{\&}$ Equal contributors *For correspondence: bcxingsubmission@163.com 
Matched tumor and non-tumor tissue specimens were obtained from 79 patients with HBV-related HCC, defined as those with B-type hepatitis surface antigen (HBsAg) positivity. Patients underwent curative surgical resection in Department of Hepatobiliary Surgery I, Peking University School of Oncology, Beijing Cancer Hospital \& Institute, Beijing, China. There were 67 males and 12 females. The median age was 49 years (range: 28-73 years). Portal vein invasion (PVI), capsule formation, tumor number and size were first estimated by preoperative imaging examinations and demonstrated during surgery. Tumor differentiation grade, according to Edmondson-Steiner criteria (Edmondson et al., 1954), was determined by postoperation histology. Patient clinicopathologic variables are summarized in Table 1 . The project was approved by the Institutional Ethics Committee.

\section{Immunohistochemical staining}

An anti-CHIP antibody (Santa Cruz Biotechnology, Inc., Santa Cruz, CA) was used for staining. Briefly, 4 $\mu \mathrm{m}$-thick sections were first mounted, deparaffinized in xylene and rehydrated in graded ethanol. Antigen retrieval was performed using a high pressure method for $3 \mathrm{~min}$. Slides were then incubated with $3 \%$ hydrogen peroxide for 10 min to eliminate endogenous peroxidase. Subsequently, slides were incubated overnight at $4^{\circ} \mathrm{C}$ with the primary antibody at a dilution of $1: 100$. Pre-immune serum at the same dilution was used as the negative control. Following washing in phosphate buffered saline (PBS), horseradish peroxidase (HRP)-labeled secondary antibody was added for an incubation of $30 \mathrm{~min}$. Diaminobenzidine was applied as a chromogen. Finally, slides were counterstained with hematoxylin.

\section{Staining evaluation}

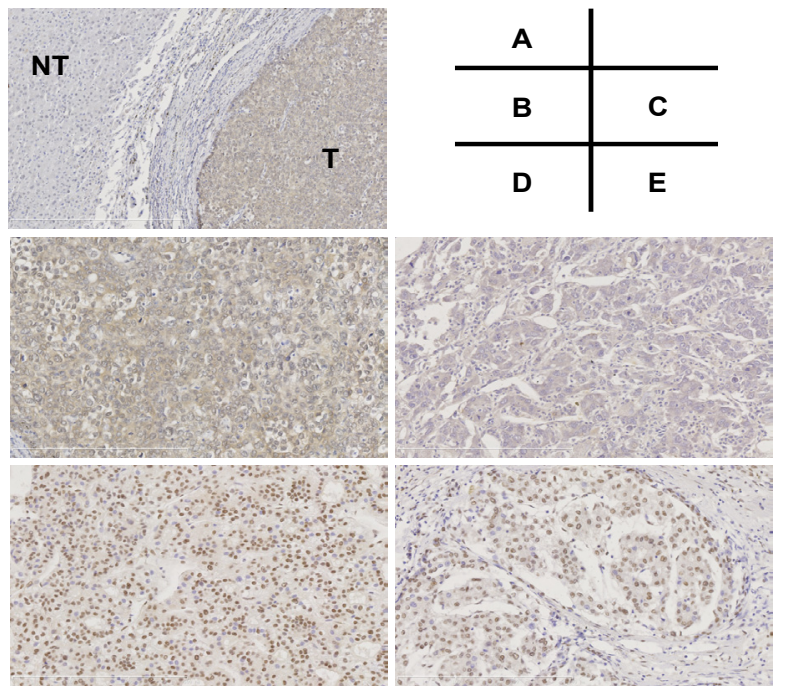

Figure 1. Expression of CHIP in Hepatocellular

Carcinoma. (A), Staining in tumor and adjacent non-tumor tissues of HCC (original magnification $\times 100$ ); (B), High cytoplasmic staining in tumor cells (original magnification $\times 200$ ); (C), Low cytoplasmic staining in tumor cells (original magnification $\times 200$ ); (D); High nuclear staining in tumor cells (original magnification $\times 200$ ); (E), Low nuclear staining in tumor cells (original magnification $\times 200$ ). CHIP, carboxyl terminus of Hsp70-interacting protein; NT, non-tumor, T tumor
Two independent pathologists who were blinded to clinicopathologic and follow-up data (Z.Y.L. and W.X.Z.) evaluated the slides, and discussed for a consensus when they were divergent. The positive signal was defined as brown coloration in tumor and non-tumor cells. Scoring of staining results was performed according to the standard previous used (Liang et al., 2013).

\section{Follow-up}

Follow-up was performed for a total of 74 patients (median follow-up period: 28 months; range: 2 to 72 months). According to the follow-up data, 42 patients have died.

\section{Statistical analyses}

The Chi-square test was applied to analyze associations between CHIP expression and clinicopathologic factors. Patient survival was calculated using Kaplan-Meier

Table 1. Associations between CHIP Expression and Clinicopathologic Variables of HBV-related HCC

\begin{tabular}{|c|c|c|c|c|c|}
\hline \multirow{2}{*}{ Variables } & & \multicolumn{4}{|c|}{ CHIP expression } \\
\hline & & $\mathrm{n}$ & High & Low & $P$ value \\
\hline \multirow[t]{2}{*}{ Sex } & Male & 67 & 29 & 38 & 0.666 \\
\hline & Female & 12 & 6 & 6 & \\
\hline \multirow[t]{2}{*}{ Age } & $\geq 60$ years & 22 & 13 & 9 & 0.100 \\
\hline & $<60$ years & 57 & 22 & 35 & \\
\hline \multirow[t]{2}{*}{ Cirrhosis } & Present & 56 & 25 & 31 & 0.861 \\
\hline & Absent & 17 & 8 & 9 & \\
\hline \multirow[t]{2}{*}{ Child-Pugh grade } & A & 66 & 31 & 35 & $0.317 *$ \\
\hline & $\mathrm{B}$ & 6 & 1 & 5 & \\
\hline \multirow[t]{2}{*}{ Serum AFP level } & $>20 \mathrm{ng} / \mathrm{ml}$ & 39 & 22 & 17 & 0.057 \\
\hline & $\leq 20 \mathrm{ng} / \mathrm{ml}$ & 30 & 10 & 20 & \\
\hline \multirow[t]{2}{*}{ Tumor number } & Solitary & 63 & 27 & 36 & 0.608 \\
\hline & Multiple & 16 & 8 & 8 & \\
\hline \multirow[t]{2}{*}{ Capsule formation } & Present & 37 & 14 & 23 & 0.162 \\
\hline & Absent & 37 & 20 & 17 & \\
\hline \multirow[t]{2}{*}{ Tumor size } & $\geq 5 \mathrm{~cm}$ & 36 & 21 & 15 & 0.022 \\
\hline & $<5 \mathrm{~cm}$ & 43 & 14 & 29 & \\
\hline \multirow[t]{2}{*}{ PVI } & Present & 21 & 13 & 8 & 0.095 \\
\hline & Absent & 52 & 21 & 31 & \\
\hline \multirow[t]{2}{*}{ Edmondson-Steiner } & Grade I-II & 59 & 26 & 33 & 0.801 \\
\hline & Grade III-IV & 19 & 9 & 10 & \\
\hline
\end{tabular}

*Chi-square test with continuity correction; CHIP, carboxyl terminus of Hsp70-interacting protein; HBV, B-type hepatitis virus; HCC, hepatocellular carcinoma; AFP, $\alpha$-fetoprotein; PVI, portal vein invasion
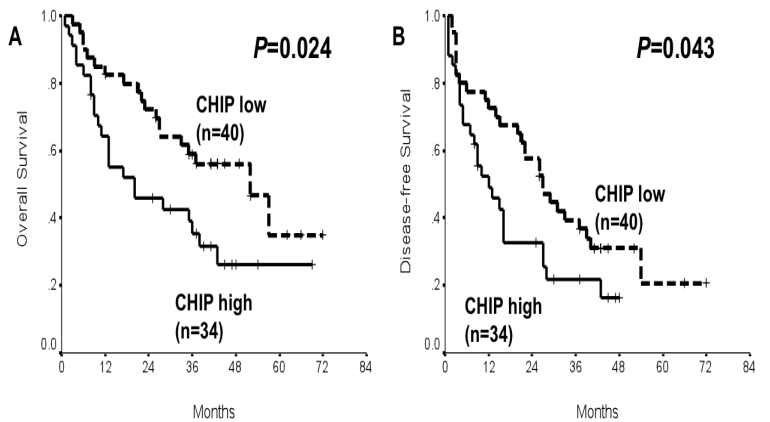

Figure 2. Influences of CHIP Expression on Survival of Hepatocellular Carcinoma. A) Overall survival; B) Disease-free survival. CHIP, carboxyl terminus of Hsp70interacting protein 
method and compared by log-rank test. Cox regression (Proportional hazard model) was used for multivariate analysis of prognostic markers. Statistical software package SPSS 11.5 (SPSS Inc, Chicago, IL) was adopted for all analyses. Statistically significant $P$ value was defined as less than 0.05 .

\section{Results}

CHIP expression and associations with clinicopathologic variables in HBV-related HCC

High expression of CHIP was present in tumor tissues from 35 patients $(44.3 \%)$ with HBV-related HCC, but absent in all the non-tumor tissues (Figure 1A). The brown
Significance of CHIP in HBV-Related Hepatocellular Carcinoma staining signal was located in both cytoplasm and nucleus of HCC cells (Figure 1B-E). High CHIP expression was significantly associated with large tumor size $(P<0.05$; Table 1), and marginally linked to elevated serum AFP level and presence of PVI $(0.05<P<0.10$; Table 1$)$. Other clinicopathologic parameters was not found to be related to CHIP expression $(P>0.05$; Table 1$)$.

Prognostic factors of $\mathrm{HBV}$-related $\mathrm{HCC}$

Univariate analysis showed that high CHIP expression was associated with poor overall and disease-free survival in HBV-related HCC $(P=0.024$ and 0.043 , respectively; Figure 2 and Table 2). In addition, Edmondson-Steiner grade, PVI, tumor number and size were also prognostic

Table 2. Univariate Analysis for Overall and Disease-free Survival of HBV-related HCC

\begin{tabular}{|c|c|c|c|c|c|c|c|c|c|}
\hline \multirow[b]{2}{*}{ Variables } & \multirow[b]{2}{*}{$\mathrm{n}$} & \multicolumn{3}{|c|}{ Overall survival } & \multirow[b]{2}{*}{$\overline{\text { P value }}$} & \multicolumn{4}{|c|}{ Disease-free survival } \\
\hline & & $\overline{1-\mathrm{yr}(\%)}$ & $3-\mathrm{yr}(\%)$ & $5-\mathrm{yr}(\%)$ & & $1-\operatorname{yr}(\%)$ & $3-\mathrm{yr}(\%)$ & $5-\mathrm{yr}(\%)$ & $\mathrm{P}$ value \\
\hline \multicolumn{10}{|l|}{ Sex } \\
\hline Male & 63 & 74.5 & 48.7 & 24.8 & \multirow{2}{*}{0.942} & 64.9 & 32.1 & 0 & \multirow{2}{*}{0.967} \\
\hline Female & 11 & 72.7 & 45.5 & 36.4 & & 45.5 & 27.3 & 27.3 & \\
\hline \multicolumn{10}{|l|}{ Age } \\
\hline$\geq 60$ years & 21 & 61.9 & 33.3 & 33.3 & \multirow[t]{2}{*}{0.155} & 61.9 & 25.8 & 25.8 & \multirow[t]{2}{*}{0.772} \\
\hline$<60$ years & 53 & 79.0 & 54.6 & 29.8 & & 62.0 & 33.6 & 11.8 & \\
\hline \multicolumn{10}{|l|}{ Cirrhosis } \\
\hline Present & 55 & 70.9 & 46.8 & 35.0 & \multirow[t]{2}{*}{0.703} & 59.9 & 29.1 & 20.6 & \multirow[t]{2}{*}{0.337} \\
\hline Absent & 16 & 81.3 & 47.9 & 23.9 & & 68.2 & 39.0 & 19.5 & \\
\hline \multicolumn{10}{|c|}{ Child-Pugh grade } \\
\hline Grade A & 64 & 73.3 & 49.7 & 31.6 & \multirow[t]{2}{*}{0.441} & 60.6 & 31.6 & 15.8 & \multirow[t]{2}{*}{0.124} \\
\hline Grade B & 6 & 66.7 & 33.3 & - & & 66.7 & 33.3 & - & \\
\hline \multicolumn{10}{|c|}{ Serum AFP level } \\
\hline$>20 \mathrm{ng} / \mathrm{ml}$ & 39 & 61.1 & 42.2 & 27.7 & \multirow[t]{2}{*}{0.056} & 53.5 & 24.4 & 13.9 & \multirow[t]{2}{*}{0.105} \\
\hline$\leq 20 \mathrm{ng} / \mathrm{ml}$ & 29 & 89.7 & 55.8 & - & & 72.4 & 40.5 & - & \\
\hline \multicolumn{10}{|c|}{ Tumor number } \\
\hline Solitary & 59 & 79.7 & 56.6 & 34.7 & \multirow[t]{2}{*}{$<0.001$} & 66.1 & 36.3 & 18.7 & \multirow[t]{2}{*}{0.011} \\
\hline Multiple & 15 & 51.9 & 11.1 & - & & 44.4 & 0 & 0 & \\
\hline \multicolumn{10}{|c|}{ Capsule formation } \\
\hline Present & 35 & 82.9 & 58.7 & 27.8 & \multirow[t]{2}{*}{0.063} & 68.2 & 31.4 & 10.6 & \multirow[t]{2}{*}{0.551} \\
\hline Absent & 36 & 63.5 & 33.3 & 33.3 & & 52.5 & 28.9 & 25.3 & \\
\hline \multicolumn{10}{|l|}{ Tumor size } \\
\hline$\geq 5 \mathrm{~cm}$ & 32 & 61.8 & 26.4 & 10.6 & 0.001 & 45.9 & 12.4 & 0 & $<0.001$ \\
\hline$<5 \mathrm{~cm}$ & 42 & 83.3 & 63.8 & 47.8 & & 73.8 & 44.4 & 33.0 & \\
\hline PVI & & & & & & & & & \\
\hline Present & 21 & 41.3 & 20.6 & 10.3 & $<0.001$ & 36.7 & 18.4 & 0 & 0.009 \\
\hline Absent & 49 & 85.7 & 59.9 & 42.7 & & 69.4 & 37.4 & 29.7 & \\
\hline Edmondson-S & ade & & & & & & & & \\
\hline I-II & 56 & 82.0 & 55.4 & 34.5 & 0.008 & 67.5 & 38.9 & 21.5 & 0.006 \\
\hline III-IV & 18 & 50.0 & 25.9 & - & & 44.4 & 6.9 & 0 & \\
\hline CHIP express & & & & & & & & & \\
\hline High & 34 & 64.2 & 35.5 & 26.3 & 0.024 & 49.1 & 21.8 & - & 0.043 \\
\hline Low & 40 & 82.5 & 58.9 & 35.0 & & 72.5 & 39.4 & 20.7 & \\
\hline
\end{tabular}

HBV, B-type hepatitis virus; HCC, hepatocellular carcinoma; yr, year; AFP, $\alpha$-fetoprotein; PVI, portal vein invasion; CHIP, carboxyl terminus of Hsp70-interacting protein

Table 3. Multivariate Analysis for Overall and Disease-free Survival of HBV-related HCC

\begin{tabular}{lccccccc}
\hline & \multicolumn{3}{c}{ Overall survival } & & \multicolumn{3}{c}{ Disease-free survival } \\
\cline { 2 - 3 } Variables & HR & $95 \%$ CI & P value & & HR & 95\%CI & P value \\
\hline Tumor number & 3.161 & $1.501-6.657$ & 0.002 & & 2.196 & $1.083-4.454$ & 0.029 \\
Edmondson-Steiner grade & 2.043 & $1.042-4.006$ & 0.038 & & 1.858 & $1.015-3.401$ & 0.045 \\
Tumor size & & & & & 1.943 & $1.018-3.709$ & 0.044 \\
CHIP expression & 1.484 & $0.731-3.015$ & 0.275 & & 1.292 & $0.698-2.391$ & 0.415 \\
\hline
\end{tabular}

HBV, B-type hepatitis virus; HCC, hepatocellular carcinoma; HR, hazard ratio; CI, confidence interval; CHIP, carboxyl terminus of Hsp70-interacting protein 
for both overall and disease-free survival $(P<0.05$; Table 2), whereas other variables were not of statistical significance $(P>0.05$; Table 2$)$. In multivariate Cox regression test, Edmondson-Steiner grade, tumor number and size were identified as independent prognostic indicators for overall and/or disease-free survival $(P<0.05$; Table 3). However, independent predictive values of CHIP expression for overall and disease-free survival were not proven $(P>0.05$; Table 3$)$.

\section{Discussion}

CHIP is an E3 ligase that is combined with heat shock protein (HSP) 70 and 90 via the tetratricopeptide repeat (TPR) domain to mediate ubiquitin-dependent protein degradation (Ballinger et al., 1999; Connell et al., 2001). Therefore, biological roles of CHIP in cells largely depend on its target proteins. So far, diverse effects of CHIP were observed in different malignancies, accompanied by identification of different targets, such as survivin, androgen receptor, NF- $\varkappa \mathrm{B} / \mathrm{p} 65$, TRAF2 and EGFR (Jang et al., 2011; Xu et al., 2011; Wang et al., 2013; Sarkar et al., 2014; Wang et al., 2014). On the other hand, clinicopathologic and prognostic implications of CHIP were also opposite in several types of cancer (Jan et al., 2011; Jang et al., 2011; Liang et al., 2013; Wang et al., 2013; Wen et al., 2013; Wang et al., 2014). Thus, CHIP seems to be a protein that functions tissue-type specifically. However, the issues have not addressed in $\mathrm{HCC}$. In view of the fact that HBV was the most frequent cause of HCC, the present work focused on HBV-related HCC. It was first found that CHIP expression was much higher in tumor tissues, in contrast to non-tumor ones (Figure 1A), indicating potential correlations of CHIP with HBV-related HCC. Surprisingly, our results showed positive nuclear staining of CHIP in tumor cells (Figure $1 \mathrm{D}-\mathrm{E})$, different with previous reports presenting its cytoplasmic location (Jan et al., 2011; Jang et al., 2011; Xu et al., 2011; Liang et al., 2013; Wang et al., 2013; Wen et al., 2013; Wang et al., 2014). The observation that HSP70, a protein that interacts with CHIP to form a complex, displayed nuclear staining in HCC tissues provides preliminary support (Effendi et al., 2010). In the future, further validations and mechanism exploration are quite needed. Then, Chi-square test revealed that high CHIP expression was significantly associated with lager tumor size, and being of marginally significant relationship with presence of PVI and high serum AFP level. The link between tumor size and aggressive phenotypes in HCC was previously established (Kaibori et al., 2010; Kim et al., 2010), whereas high serum AFP level and formation of PVI were also proven to contribute to progression and recurrence of the malignancy (Peng et al., 2004; Choi et al., 2011). Therefore, CHIP seems to be involved in malignant biological behaviors and serve as an oncogene in HBV-related HCC. Of course, investigations on the detailed mechanisms are expected.

Similarly, the influence of CHIP on prognosis of a line of cancers was also suggested to be divergent (Jan et al., 2011; Liang et al., 2013; Wang et al., 2013; Wen et al., 2013; Wang et al., 2014), because CHIP expression was associated with satisfactory outcome in gastric, pancreatic and breast cancers (Jan et al., 2011; Wang et al., 2013; Wang et al., 2014), while related to gloomy survival in gallbladder and esophageal cancers (Liang et al., 2013; Wen et al., 2013). However, no data derived from HCC. In the current investigation, we found that high expression of CHIP was predictive for poor survival in univariate analysis, together with some conventional clinicopathologic variables. Considering aforementioned results that CHIP expression was associated with malignant phenotypes, its prognostic implication might be easily understood. Therefore, CHIP might act as a potential biomarker for unfavorable prognosis of HBVrelated HCC. It is a pity that CHIP was not proven to be an independent prognostic factor in multivariate analysis. Thus, the value of this protein in prognosis prediction of HBV-related HCC remains to be evaluated in large-scale prospective studies.

Taken together, our data demonstrated that CHIP is overexpressed in HBV-related HCC and associated with unfavorable biological behaviors as well as poor prognosis.

\section{Acknowledgements}

This work was supported by the National Natural Science Foundation of China (81301845).

\section{References}

Ballinger CA, Connell P, Wu Y, et al (1999). Identification of CHIP, a novel tetratricopeptide repeat-containing protein that interacts with heat shock proteins and negatively regulates chaperone functions. Mol Cell Biol, 19, 4535-45.

Choi KK, Kim SH, Choi SB, et al (2011). Portal venous invasion: the single most independent risk factor for immediate postoperative recurrence of hepatocellular carcinoma. $J$ Gastroenterol Hepatol, 26, 1646-51.

Connell P, Ballinger CA, Jiang J, et al (2001). The co-chaperone CHIP regulates protein triage decisions mediated by heatshock proteins. Nat Cell Biol, 3, 93-6.

Edmondson HA, Steiner PE (1954). Primary carcinoma of the liver: a study of 100 cases among 48,900 necropsies. Cancer, 7, 462-503.

Effendi K, Sakamoto M (2010). Molecular pathology in early hepatocarcinogenesis. Oncology, 78, 157-60.

El-Serag HB (2011). Hepatocellular carcinoma. $N$ Engl J Med, 365, 1118-27.

Jan CI, Yu CC, Hung MC, et al (2011). Tid1, CHIP and ErbB2 interactions and their prognostic implications for breast cancer patients. J Pathol, 225, 424-37.

Jang KW, Lee KH, Kim SH, et al (2011). Ubiquitin ligase CHIP induces TRAF2 proteasomal degradation and NF- $x \mathrm{~B}$ inactivation to regulate breast cancer cell invasion. J Cell Biochem, 112, 3612-20.

Kaibori M, Ishizaki M, Matsui K, Kwon AH (2010). Predictors of microvascular invasion before hepatectomy for hepatocellular carcinoma. J Surg Oncol, 102, 462-8.

Kim SJ, Lee KK, Kim DG (2010). Tumor size predicts the biological behavior and influence of operative modalities in hepatocellular carcinoma. Hepatogastroenterology, 57, 121-6.

Liang ZL, Kim M, Huang SM, Lee HJ, Kim JM (2013). Expression of carboxyl terminus of Hsp70-interacting 
protein (CHIP) indicates poor prognosis in human gallbladder carcinoma. Oncol Lett, 5, 813-8.

Mínguez B, Lachenmayer A (2011). Diagnostic and prognostic molecular markers in hepatocellular carcinoma. Dis Markers, 31, 181-90.

Peng SY, Chen WJ, Lai PL, et al (2004). High alpha-fetoprotein level correlates with high stage, early recurrence and poor prognosis of hepatocellular carcinoma: significance of hepatitis virus infection, age, 553 and beta-catenin mutations. Int J Cancer, 112, 44-50.

Parkin DM, Pisani P, Ferlay J (1993). Estimates of the worldwide incidence of eighteen major cancers in 1985. Int J Cancer, 54, 594-606.

Parkin DM, Bray F, Ferlay J, Pisani P (2005). Global cancer statistics, 2002. CA Cancer J Clin, 55, 74-108.

Pisani P, Parkin DM, Bray F, Ferlay J (1999). Estimates of the worldwide mortality from 25 cancers in 1990. Int J Cancer, 83, 18-29.

Poon RT, Fan ST, Lo CM, et al (2001). Improving survival results after resection of hepatocellular carcinoma: a prospective study of 377 patients over 10 years. Ann Surg, 234, 63-70.

Sarkar S, Brautigan DL, Parsons SJ, Larner JM (2014). Androgen receptor degradation by the E3 ligase CHIP modulates mitotic arrest in prostate cancer cells. Oncogene, 33, 26-33.

Shimozawa N, Hanazaki K (2004). Longterm prognosis after hepatic resection for small hepatocellular carcinoma. $\mathrm{J} \mathrm{Am}$ Coll Surg, 198, 356-65.

Tandon P, Garcia-Tsao G (2009). Prognostic indicators in hepatocellular carcinoma: a systematic review of 72 studies. Liver Int 29, 502-10.

Verhoef C, de Man RA, Zondervan PE, et al (2004). Good outcomes after resection of large hepatocellular carcinoma in the non-cirrhotic liver. Dig Surg, 21, 380-6.

Wang S, Wu X, Zhang J, et al (2013). CHIP functions as a novel suppressor of tumour angiogenesis with prognostic significance in human gastric cancer. Gut, 62, 496-508.

Wang T, Yang J, Xu J, et al (2014). CHIP is a novel tumor suppressor in pancreatic cancer through targeting EGFR. Oncotarget, 5, 1969-86.

Wen J, Luo KJ, Hu Y, Yang H, Fu JH (2013). Metastatic lymph node CHIP expression is a potential prognostic marker for resected esophageal squamous cell carcinoma patients. Ann Surg Oncol, 20, 1668-75.

Xu T, Zhou Q, Zhou J, et al (2011). Carboxyl terminus of Hsp70interacting protein (CHIP) contributes to human glioma oncogenesis. Cancer Sci, 102, 959-66. 